Case Report

\title{
Should de Winter T-Wave Electrocardiography Pattern Be Treated as ST-Segment Elevation Myocardial Infarction Equivalent with Consequent Reperfusion? A Dilemmatic Experience in Rural Area of Indonesia
}

\author{
Raymond Pranata $\mathbb{D}^{1,2}$ Ian Huang $\mathbb{D}^{1},{ }^{1}$ and Vito Damay $\mathbb{D}^{1,3}$ \\ ${ }^{1}$ Faculty of Medicine, Universitas Pelita Harapan, Tangerang, Banten, Indonesia \\ ${ }^{2}$ Tabanan General Hospital, Tabanan, Bali, Indonesia \\ ${ }^{3}$ Siloam Hospitals Lippo Village, Tangerang, Banten, Indonesia
}

Correspondence should be addressed to Vito Damay; vito.damay@uph.edu

Received 21 January 2018; Revised 1 March 2018; Accepted 13 March 2018; Published 31 March 2018

Academic Editor: Hajime Kataoka

\begin{abstract}
Copyright (c) 2018 Raymond Pranata et al. This is an open access article distributed under the Creative Commons Attribution License, which permits unrestricted use, distribution, and reproduction in any medium, provided the original work is properly cited.
\end{abstract}

\begin{abstract}
Background. Although de Winter T-wave electrocardiography pattern is rare, it signifies proximal left anterior descending artery occlusion and is often unrecognized by physicians. The aim of this case report was to highlight the dilemma in the management of a patient with de Winter T-wave pattern in the hospital without interventional cardiology facility. Case Presentation. A 65-yearold male presented with typical chest pain since 2 hours before admission, and ECG showed sinus rhythm of $57 \mathrm{bpm}$ and $>1 \mathrm{~mm}$ upsloping ST depression with symmetric tall $\mathrm{T}$ in lead $\mathrm{V}_{2-3}$ characteristic of de Winter T-wave ECG pattern. He was given dual antiplatelet therapy, nitrate, statin, and anticoagulant. He refused referral to interventional cardiology available hospital. 3 hours after admission, the electrocardiography transformed into Q-waves consistent with final stages of acute STEMI and ST-segment elevation that barely meets the threshold in the guideline, and thrombolytic was administered and successful. There is a suggestion that de Winter T-wave electrocardiography should be treated as ST-segment myocardial infarction equivalent and should undergo coronary angiography; however, not every hospital has the luxury of interventional cardiology facility. The other modality for reperfusion is thrombolysis; however, without a clear guideline and scarcity of study, we prefer to resort to conservative treatment. "Fortunately," transformation into ST-segment elevation helps us to determine the course of action which is reperfusion using thrombolytic. Conclusions. de Winter T-wave ECG pattern is not mentioned in any guidelines regarding acute coronary syndromes, and there are no clear recommendations. Physicians in rural area without interventional cardiology facility face a dilemma with the lack of evidence-based guideline. Fibrinolytic may be appropriate in those without contraindications with strong chest pain consistent with acute coronary occlusion, less than 3 hours of symptoms, and convincing de Winter T-wave ECG pattern for a rural non-PCI hospital far away from PCI capable hospital.
\end{abstract}

\section{Background}

In 2008, de Winter et al. reported an electrocardiography (ECG) pattern that is found in about $2 \%$ of patients with proximal left anterior descending artery occlusion (acute anterior myocardial infarction) and is often unrecognized by physicians $[1,2]$. Although de Winter T-wave ECG pattern was suggested to be managed as STEMI equivalent [3], it was not mentioned in recent guidelines by the European Heart Society (ESC) and the American Heart Association (AHA) [4, 5]. Hence, a question arises regarding whether or not this pattern be treated with fibrinolytic in an area where cardiac catheter laboratory (cath lab) is not readily available. The aim of this case report was to highlight the dilemma in the management of a patient with de Winter T-wave pattern in hospital without interventional cardiology facility (non-PCI hospital). 


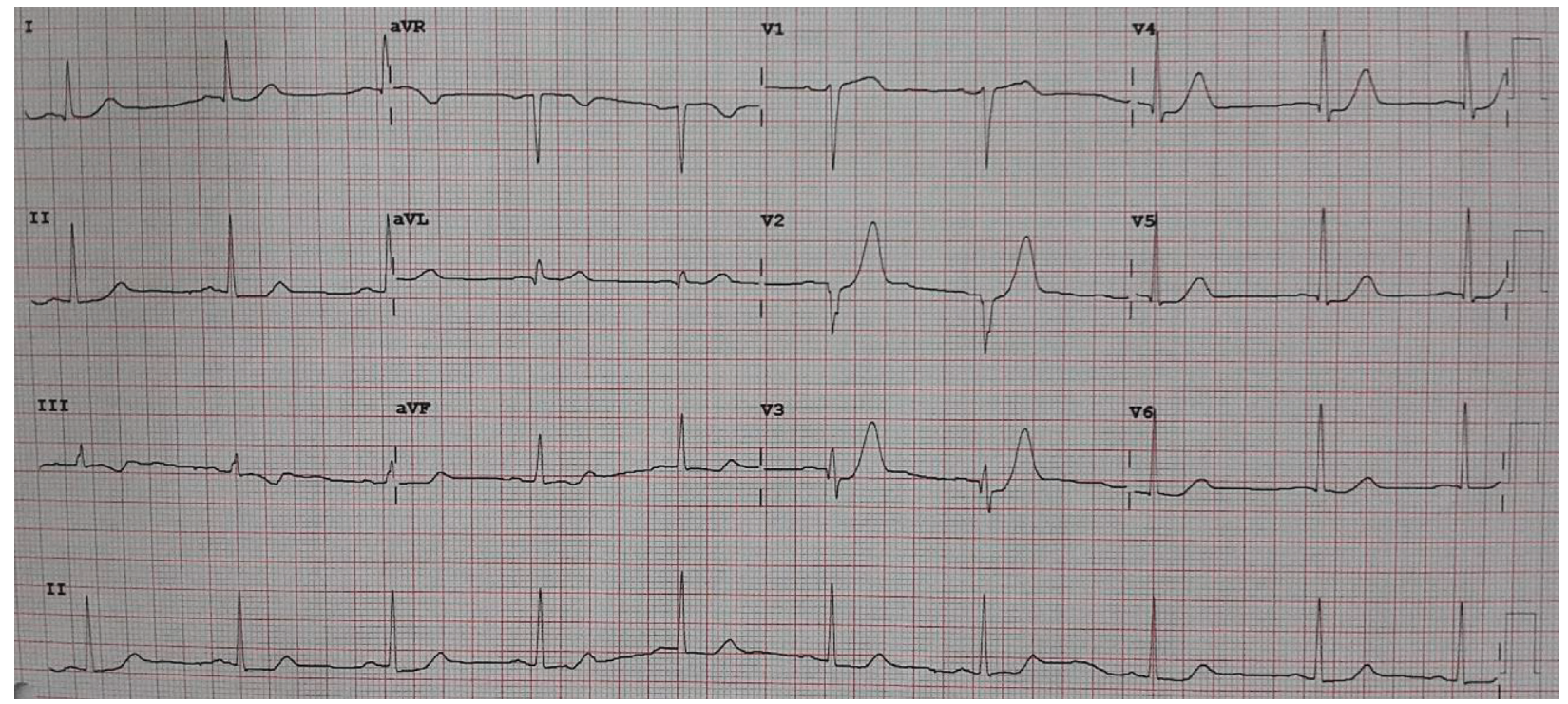

FIGURE 1: Initial ECG showing > 1 mm upsloping ST depression with symmetric tall T in lead $\mathrm{V}_{2-6}$ characteristic of de Winter ECG pattern.

\section{Case Presentation}

A 65-year-old male presented to the emergency department with typical chest pain at rest since 2 hours before admission after eating. The patient denied shortness of breath and previous episode of chest pain before admission. He had a past medical history of poorly controlled hypertension and was taking captopril. History of diabetes, smoking, and previous stroke or myocardial infarction was denied. On physical examination, blood pressure was $150 / 90 \mathrm{mmHg}$, heart rate was $57 \mathrm{x} /$ minute, and respiratory rate was $20 \mathrm{x} / \mathrm{minute}$, and the cardiopulmonary examination was within normal limits. ECG showed sinus rhythm of $57 \mathrm{bpm}$ and $>1 \mathrm{~mm}$ upsloping ST depression with symmetric tall $\mathrm{T}$ in lead $\mathrm{V}_{2-3}$ characteristic of de Winter T-wave ECG pattern (Figure 1). Routine blood examination, AST/ALT, BUN, and serum creatinine were within normal limits. Sodium was $137 \mathrm{mmol} / \mathrm{L}$, and potassium was $3.4 \mathrm{mmol} / \mathrm{L}$ denoting that tall $\mathrm{T}$ was not due to hyperkalemia. Troponin at the 2 nd hour after onset was negative. The patient was diagnosed with acute coronary syndrome with suspected de Winter T-wave ECG pattern. Aspirin $160 \mathrm{mg}$, clopidogrel $300 \mathrm{mg}$, sublingual isosorbide dinitrate, simvastatin $20 \mathrm{mg}$ (atorvastatin was not available), and enoxaparin $0.4 \mathrm{mg}$ subcutaneous were administered. The patient refused referral to the interventional cardiology capable hospital due to distance and socioeconomic causes. Repeat ECG was done 3 hours later showing Q-waves developing in $\mathrm{V}_{2-4}$, consistent with the final stages of acute STEMI accompanied with ST-segment elevation in lead $\mathrm{V}_{2-4}$ which barely meets the $2 \mathrm{~mm}$ threshold for those precordial leads as per the guidelines (Figure 2). Elevated troponin at the 5 th hour confirmed the presence of myocardial infarction. The patient still experienced sustained and severe chest pain although not as intense as initial presentation. Streptokinase (1.5 million units) was administered, and chest pain subsided with a resolution of ST-segment elevation (Figure 3). The patient was discharged on the 5th day after admission.

\section{Discussion}

The de Winter T-wave ECG pattern may signify proximal left anterior descending artery occlusion and was suggested as STsegment elevation myocardial infarction (STEMI) equivalent [1-3]. The de Winter T-wave ECG pattern was also shown to have positive predictive values of $95.2 \%$ (95\% confidence interval: $76.2-99.9 \%), 100 \%$ (69.2-100.0\%), and 100\% (51.7-100\%) for acute coronary occlusions in the three diagnostic studies [6]. Verouden et al. also reported that approximately $50 \%$ of those with de Winter T-wave ECG pattern had a wraparound LAD which is associated with larger area of ischemia [2]. However, despite these findings, de Winter T-wave ECG pattern is yet to be included in ESC guidelines for management of acute coronary syndromes in both persistent (2017) or without persistent STelevation (2015) and ACC/AHA guidelines regarding STEMI (2013) and NSTEMI (2014) $[4,5]$.

Ideally, the presence of de Winter T-wave ECG should be treated as urgent as STEMI with catheter lab activation for coronary angiography and possible stenting. This was not possible in our case due to a limited facility in the region [3]. Thrombolysis was initially avoided because de Winter T-wave ECG is currently not an indication for fibrinolysis even in latest guidelines, and there was no clear-cut evidence of acute coronary occlusion. The lack of the evidence-based guidelines for the aforementioned condition compelled us to choose dual antiplatelet therapy and anticoagulant for the initial mainstay of therapy. Evolution from suspected de Winter T-wave pattern to the final stage of acute STEMI was observed three hours after admission. This helped us in ushering the direction of management, as the guideline for STEMI is straightforward in terms of reperfusion. Thrombolysis was initiated, and repeat ECG showed resolution of chest pain and elevated ST-Segment which proves the acute coronary occlusion (anterior myocardial infarction).

Whether de Winter T-wave ECG pattern can evolve into STEMI is debatable, and there are arguments that de Winter 


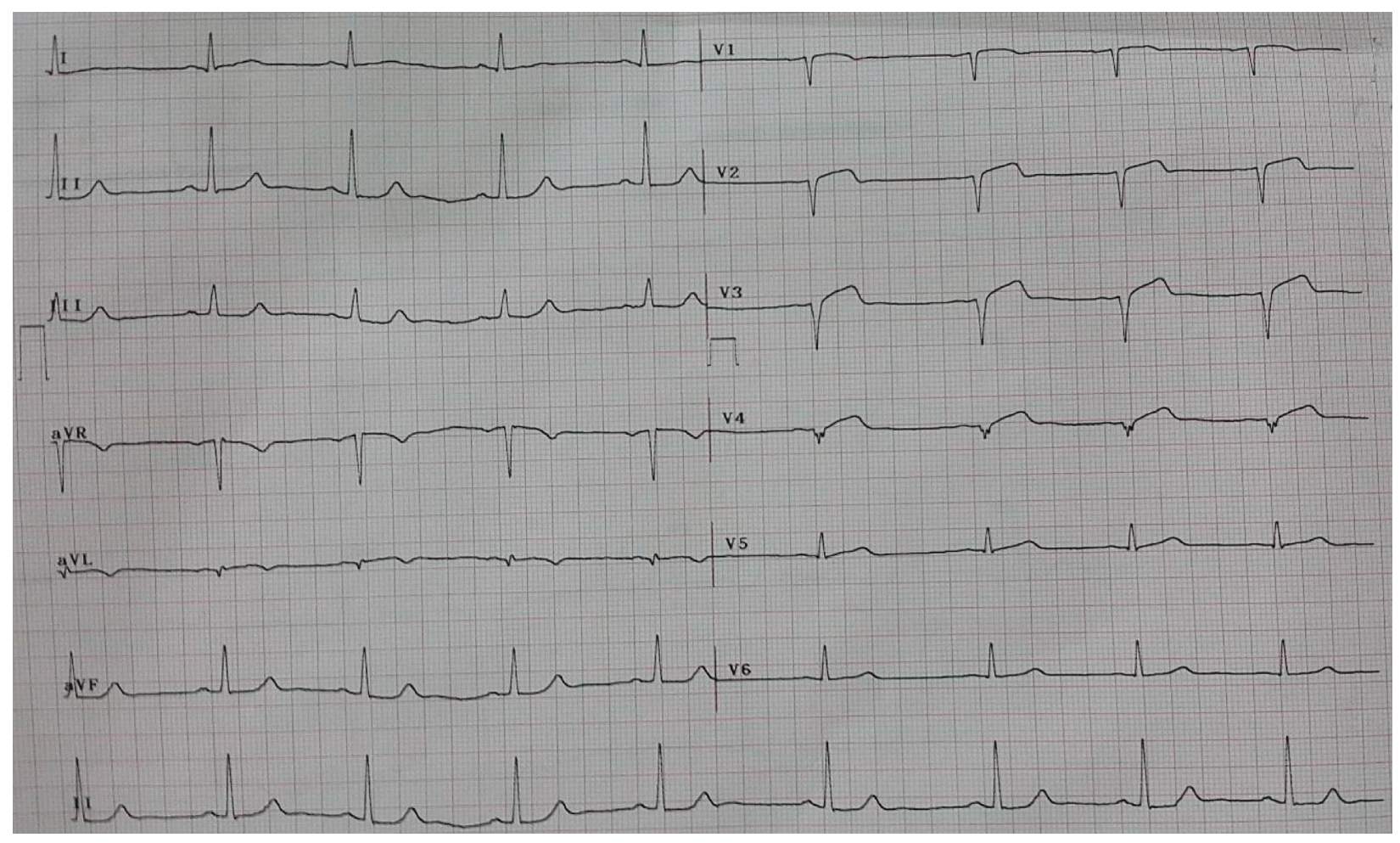

FIGURE 2: ECG showing progression into ST-segment elevation myocardial infarction.

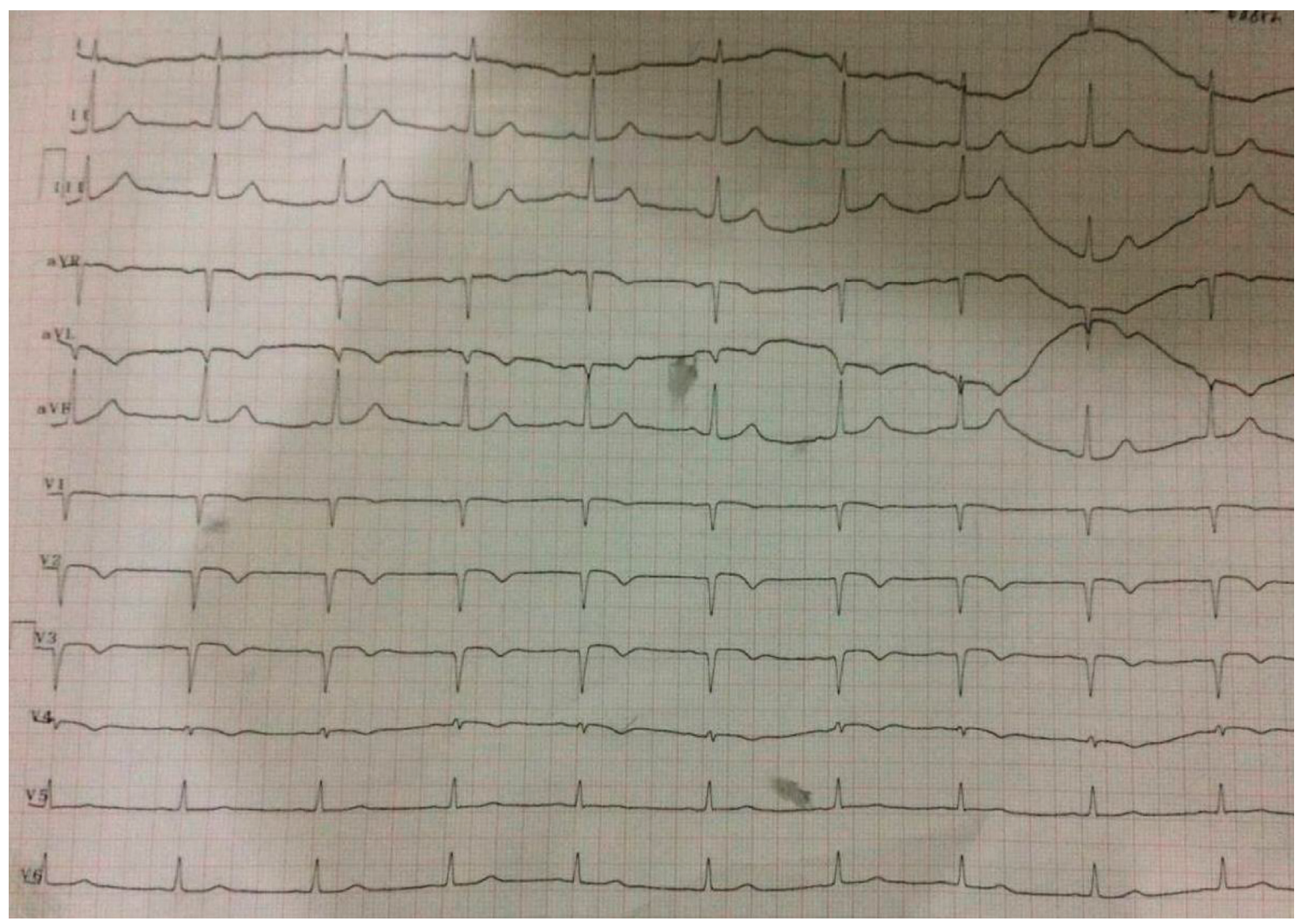

FIGURE 3: ECG after fibrinolysis showing resolution of ST-segment elevation. 
T-wave pattern is a part of STEMI evolution which was impeded with aggressive antithrombotic/antiplatelet therapy [7]. Another opinion states that ECG evolution into STEMI was actually indicative of hyperacute $\mathrm{T}$-wave instead of de Winter T-wave ECG pattern, in which the latter usually progresses directly to sign of transmural infarction on ECG $[3,8]$. A more diplomatic view was the categorization of de Winter T-wave ECG pattern into those 2 debated groups, respectively [9]. The presence of Q-waves in our patient with ST-segment elevation which barely meets $2 \mathrm{~mm}$ suggests transformation into final stages of acute STEMI; hence, our patient showed both evolution into STEMI and signs of transmural infarction (Q-wave).

Regardless of the debate, the most important issue is to recognize this ECG pattern and prevent the delay in management. Delay leads to a higher total ischemic time which is related to higher mortality in STEMI; however, whether the same applies to de Winter T-wave ECG is unclear [10].

\section{Conclusions}

The de Winter T-wave ECG pattern is not mentioned in any guidelines regarding acute coronary syndromes, and there are no clear recommendations. Physicians in rural area without interventional cardiology facility face a dilemma with the lack of evidence-based guideline and prefer to resort to a conservative strategy rather than potentially doing harm. Such approach may or may not lead to the best possible outcome but is the only choice in the middle of scarcity. Fibrinolysis may be considered in a young patient (without usual contraindications) who arrives with strong chest pain consistent with acute coronary occlusion, less than 3 hours of symptoms, and with convincing de Winter T-wave ECG pattern (especially if a prior baseline ECG is available for comparison) for a rural non-PCI hospital far away from PCI capable hospital. Discussion with the interventional cardiologist at the PCI hospital whenever possible should also be done in these challenging cases to optimize real-time decision-making.

\section{Abbreviations}

AST: $\quad$ Aspartate aminotransferase

ALT: $\quad$ Alanine aminotransferase

BUN: Blood urea nitrogen

ECG: $\quad$ Electrocardiogram

STEMI: ST-segment elevation myocardial infarction

NSTEMI: Non-ST-segment elevation myocardial infarction.

\section{Consent}

Written informed consent was obtained from the patient for publication of this case report and any accompanying images.

\section{Conflicts of Interest}

The authors declare that they have no conflicts of interest.

\section{Authors' Contributions}

Raymond Pranata admitted, evaluated, and treated the patient. He also planned and drafted the manuscript. Ian Huang performed extensive research on the topic and helped in giving another point of view based on theory and experience. Vito Damay supervised and gave expert advice regarding the manuscript. All authors read and approved the final manuscript.

\section{References}

[1] R. J. de Winter, N. J. Verouden, H. J. Wellens, and A. A. Wilde, "A new ECG sign of proximal LAD occlusion," New England Journal of Medicine, vol. 359, no. 19, pp. 2071-2073, 2008.

[2] N. J. Verouden, K. T. Koch, R. J. Peters et al., "Persistent precordial "hyperacute" T-waves signify proximal left anterior descending artery occlusion," Heart, vol. 95, no. 20, pp. 1701-1706, 2009.

[3] I. C. Rokos, W. J. French, A. Mattu et al., "Appropriate cardiac cath lab activation: optimizing electrocardiogram interpretation and clinical decision-making for acute ST-elevation myocardial infarction," American Heart Journal, vol. 160, no. 6, pp. 9951003, 2010.

[4] B. Ibanez, S. James, S. Agewall et al., "2017 ESC guidelines for the management of acute myocardial infarction in patients presenting with ST-segment elevation," European Heart Journal, vol. 39, pp. 119-177, 2017.

[5] P. T. O'Gara, F. G. Kushner, D. D. Ascheim et al., "ACCF/AHA guideline for the management of ST-elevation myocardial infarction: a report of the American College of Cardiology Foundation/American Heart Association task force on practice guidelines," Circulation, vol. 127, no. 4, pp. e362-e425, 2013.

[6] N. P. Morris and R. Body, "The de winter ECG pattern: morphology and accuracy for diagnosing acute coronary occlusion: systematic review," European Journal of Emergency Medicine, vol. 24, no. 4, pp. 236-242, 2017.

[7] M. F. Sala, A. B. de Luna, A. C. López, and J. Garcia-Níebla, "The "De Winter Pattern" can progress to ST-segment elevation acute coronary syndrome," Revista Española de Cardiología, vol. 68, no. 11, pp. 1042-1043, 2015.

[8] I. Stankovic, I. Ilic, M. Panic, A. Vlahovic-Stipac, B. Putnikovic, and A. N. Neskovic, "The absence of the ST-segment elevation in acute coronary artery thrombosis: what does not fit, the patient or the explanation?," Journal of Electrocardiology, vol. 44, no. 1, pp. 7-10, 2011.

[9] Y. T. Zhao, L. Wang, and Z. Yi, "Evolvement to the de Winter electrocardiographic pattern," American Journal of Emergency Medicine, vol. 34, no. 2, pp. 307-337, 2016.

[10] A. E. Denktas, H. V. Anderson, J. McCarthy, and R. W. Smalling, "Total ischemic time: the correct focus of attention for optimal ST-segment elevation myocardial infarction care," JACC: Cardiovascular Interventions, vol. 4, no. 6, pp. 599-604, 2011. 


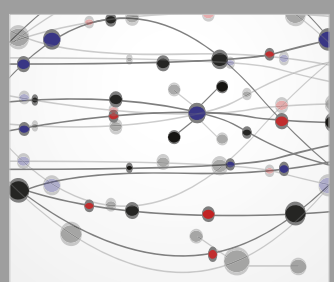

The Scientific World Journal
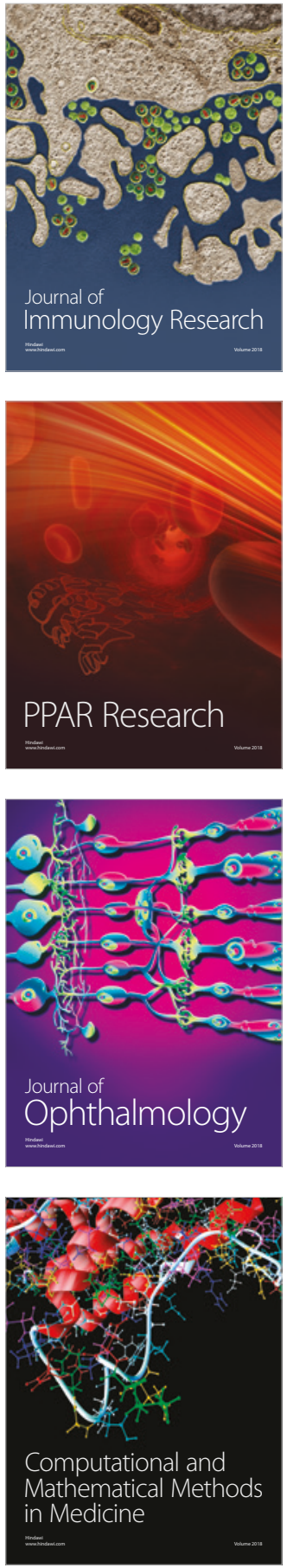

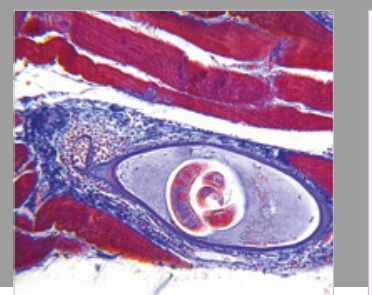

Gastroenterology Research and Practice

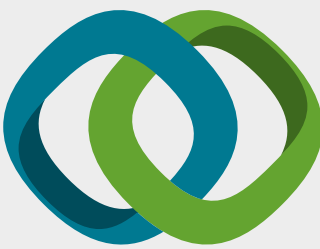

\section{Hindawi}

Submit your manuscripts at

www.hindawi.com
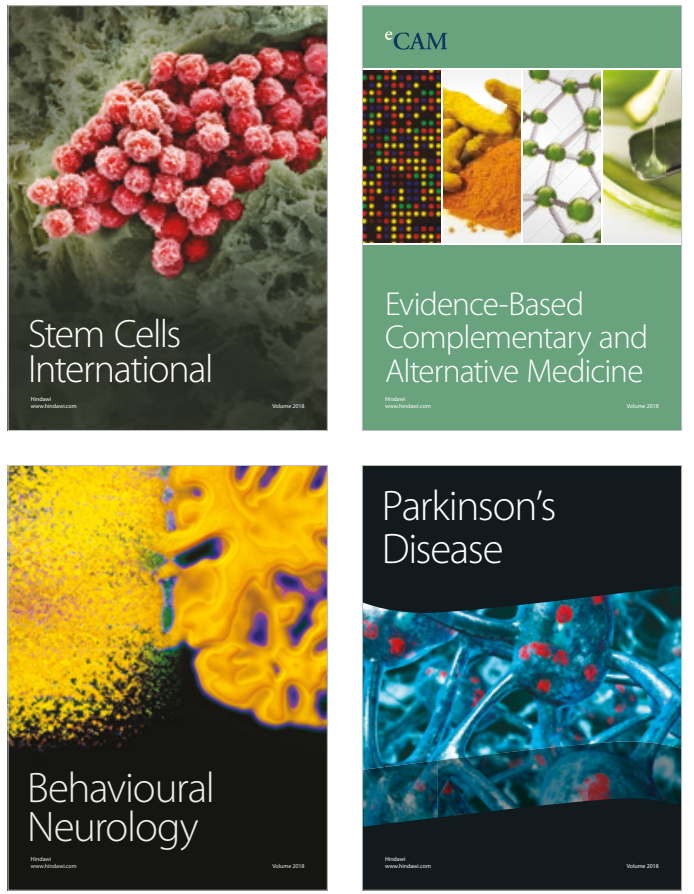

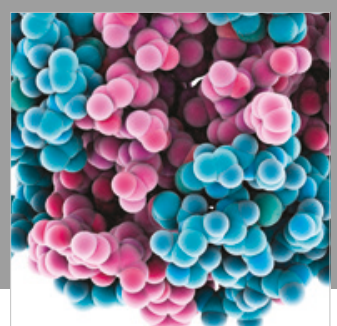

ournal of

Diabetes Research

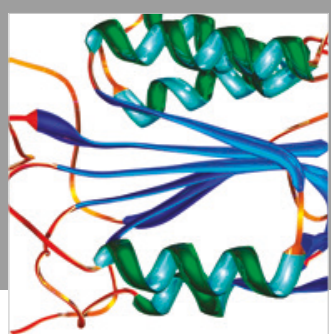

Disease Markers
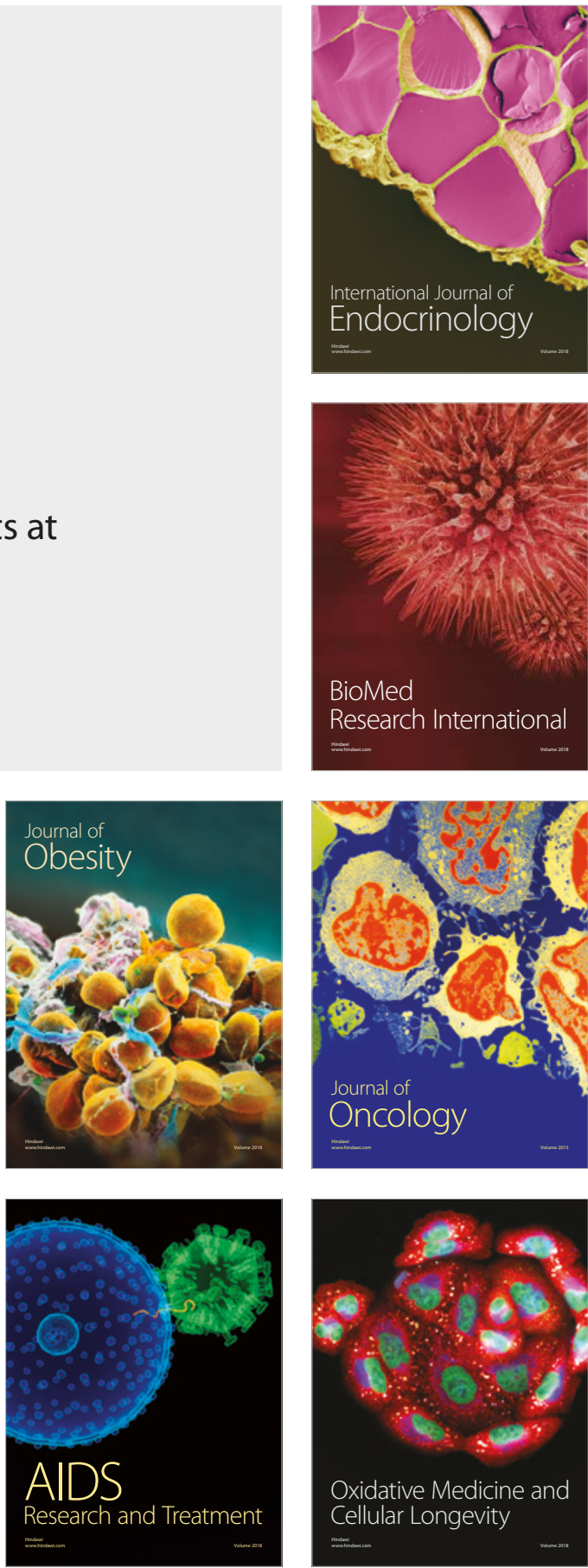